\title{
Opportunities to restore neurological function in stroke
}

\author{
Argye E Hillis
}

Why does Nature Clinical Practice Neurology publish so many articles on stroke, and why have we decided to focus on cerebrovascular disease this month? As Cheryl Bushnell reminds us in a Review in this issue, one in five women and one in six men will have a stroke at some point in their lives, and stroke can have a devastating impact on the lives of people of all ages, races and cultures.

For over a decade, management of ischemic stroke has focused on treatment during the first $3 \mathrm{~h}$ after onset, regardless of the stroke subtype. This approach derives from the fact that intravenous tissue plasminogen activator administered within $3 \mathrm{~h}$ of onset is the only FDA-approved treatment for acute ischemic stroke. This treatment is, however, useful for only $3-8 \%$ of stroke patients, so there is an urgent need for new approaches to prevention, acute treatment and rehabilitation to improve outcomes in the remaining cases.

There are opportunities to limit damage to the brain and to restore function many hours or even days after stroke onset. A Research Highlight in this issue describes a recent pilot trial that indicates that minocycline can drastically improve outcome if administered up to $48 \mathrm{~h}$ after stroke onset.

It is also possible to intervene before stroke occurs. As discussed in a Practice Point by Liao, and in a Review by Sanossian and Ovbiagele, statins are not only effective for primary stroke prevention, but can also prevent worsening of neurological status after acute stroke, and prevent stroke recurrence and new cardiovascular events. Some methods of neuroprotection might be effective only if administered before the onset of ischemia, so it would be desirable to identify and 'pre-treat' individuals who are at high immediate risk of experiencing stroke, such as those who have had a transient ischemic attack, or those undergoing surgical ...stroke

can have a devastating impact on the lives of people of all ages, races and cultures

AE Hillis is an Advisory Board member of Nature Clinical Practice Neurology.

\section{Competing interests}

The author declared no competing interests.

www.nature.com/clinicalpractice doi:10.1038/ncpneuro0708 procedures that carry a high stroke risk. A recent study highlighted by Prabhakaran in this issue showed that patients with transient ischemic attack who had acute ischemic abnormalities on diffusion-weighted imaging were more likely than those with normal imaging results to have a stroke within a year.

We also need to identify treatments targeted at specific stroke subtypes. A Review by Caplan and a Practice Point by Dafer and Biller in this issue highlight the lack of evidence regarding the suitability of anticoagulants or aspirin for preventing stroke recurrence or death in patients with cervical or intracranial dissections. In a Practice Point, Ichord discusses new observational data on stroke recurrence in cerebral sinovenous thrombosis, the most common cause of stroke in children. One risk factor for stroke recurrence in this condition is the Gly20210Ala mutation in the prothrombin gene, a finding that indicates that treatments should be tailored to genotype as well as stroke etiology. Bushnell discusses how the effects of estrogen on stroke outcome might be modulated by the APOE4 genotype, and she calls for basic research into the effects on the neurovascular unit of age, sex, vascular risk factors, endogenous sex hormone levels and genetic polymorphisms.

Future basic and clinical research should focus on the development of therapies that will help a higher proportion of people, including treatments with large effect sizes in particular patient subgroups and interventions that are effective hours to days after stroke. It is also important to identify recovery mechanisms that can be exploited to restore motor and cognitive function after stroke. This journal's emphasis on stroke reflects the opportunities that are at hand to preserve or restore function and dignity in a large number of men, women and children. 